\title{
CANCIONES INFANTILES PARA APRENDER MATEMÁTICAS
}

\author{
CHILDREN'S SONGS TO LEARN MATH
}

\section{Manuel Barrantes López, Cristina Isabel Calvino Haut, María Consuelo Barrantes Masot, Víctor Zamora Rodríguez}

\author{
Universidad de Extremadura
}

\author{
Correspondencia Manuel Barrantes López \\ Correo: barrante@unex.es \\ Recibido: 25-08-2019 Aceptado:08/06/2020 \\ DOI: 10.17398/0213-9529.40.1.103
}

\section{RESUMEN}

La Música es un recurso para la enseñanza y el aprendizaje de los contenidos matemáticos para la etapa de Educación Infantil (E.I.) y los primeros cursos de Educación Primaria (E.P.). En este estudio se ha realizado una exhaustiva búsqueda, selección y categorización de canciones que son una potente herramienta para facilitar el trabajo de los docentes en la enseñanza y aprendizaje de los contenidos matemáticos. El producto obtenido de tal investigación son 98 canciones distribuidas en 8 categorías de análisis, de acuerdo con los currículos de E.I. y E.P,que se presentan en fichas con apartados como: código de identificación, etapa y curso, categoría correspondiente, objetivos, letra y partitura, y un apartado con sugerencias y actividades para trabajar la canción en el aula. Todas las canciones están incluidas en una página web en la que se pueden consultar o descargar dichas fichas de las canciones.

Palabras clave: Música; Matemáticas; canciones; Infantil; Primaria.

\begin{abstract}
Music is a resource for teaching and learning mathematics contentstopre-school and the first levels of Primary Education.In this studyan exhaustive search, selection and categorization of songsis carried. It is a powerful tool to make easy the teacher's job in the learning and education mathematics contents. The result obtained fromthis investigation are 98 songs that have been distributed in 8 categories of analysis, in agreement with curriculum of pre-schooland primary education, They are categorized in worksheets with sections such as: code of identification, period and course, corresponding category, goals, lyric and score, and a section with suggestions and activities to work the song in the classroom.Allsongsare included in a web page where theycanbe checked or downloaded.
\end{abstract}

Keys words: Music; mathematics; songs; Pre-school; Primary.

\footnotetext{
Conflicto de intereses I Conflicts of Interest: Los autores no declaran conflicto de intereses. Sección / Section: Artículos originales Editor de Sección / Edited by: Luis Manuel Casas García / Universidad de Extremadura, España
} 


\section{INTRODUCCIÓN}

\section{Importancia de la música en la enseñanza y el aprendizaje de las matemáticas}

La música ejerce una notable influencia en la enseñanza y el aprendizaje, debido a que la educación musical contribuye al desarrollo de la inteligencia de los alumnos. A través de ella el alumno puede potenciar y adquirir conceptos propios de cualquier otra disciplina, dado que se trata de una Ciencia que estructura la forma de pensar y logra verdaderos aprendizajes significativos, sea cual sea la materia a la que se aplique.

Numerosas investigaciones que han tenido lugar en el ámbito educativo con disciplinas como Biología, Pedagogía, Fisiología, Psicología y la Música en sí misma, señalan la necesidad de incorporar la educación musical para que el individuo consiga una formación integral en todas sus facultades y no exclusivamente centrada en mejorar las habilidades musicales.

Para Casas (2001), los distintos aprendizajes y las diversas formas que tiene el alumno para acceder al conocimiento, así como la dimensión afectiva, cognitiva y psicomotriz, pueden mejorar significativamente si el alumno se inicia de forma temprana en la educación musical. Por tanto, la música no sólo alegra y reconforta al compositor, como al oyente, sino que también desarrolla la inteligencia, la creatividad e imaginación, la sensibilidad, y en general mejora el aprendizaje llegando incluso a aumentar el nivel académico (Pascual, 2006; Levitin, 2011).

Es evidente la relación tan estrecha que existe entre la Música y las Matemáticas, dos disciplinas muy diferentes, pero a su vez muy relacionadas, aunque en pocas ocasiones se hace explícita esa conexión sobre las cualidades y características que tienen en común la una con la otra. Esta relación es lógica debido a que en las actividades musicales siempre se pueden encontrar modelos matemáticos (Montiel, 2017).

También para Iturbe (2012), en la teoría de la Música encontramos conceptos propios de la Matemática; por ejemplo, en la lectura de una partitura, donde observamos que el pentagrama se compone por cinco líneas y cuatro espacios en los cuales se colocan las notas musicales que se representan mediante figuras, y pueden reducirse mediante fracciones según su valor. Los acordes también son la suma de sonidos, y los intervalos se entienden como la distancia que existe entre éstos. De igual modo, el ritmo y el sonido son cuantificables y todas las cualidades del sonido (frecuencia, timbre, altura, duración, amplitud y periodo) pueden medirse, observarse y reducirse a través de números. Rodríguez (2011) hace evidente la unión entre la teoría de ambas disciplinas manifestando que la música tiene mucho de orden y la matemática mucho de sensibilidad, belleza y armonía (p. 43).

En diferentes países se han empezado ya a realizar actividades y a elaborar materiales para interrelacionar dichas materias en la enseñanza escolar. (Casals, Carrillo y González, 2014; Hughes, 2014; Sanders, 2012).

Por otra parte, diferentes investigaciones (Alsina, 2012; Iturbe, 2012; Novo, 2013; Chao, Mato y López 2015) ponen de manifiesto que la música y de forma específica las canciones, son un medio muy adecuado para desarrollar la competencia matemática en los alumnos, fundamentalmente en las primeras edades. Mato, Chao, y Chao, (2019) realizan actividades musicales, durante dos meses, relacionadas con contenidos matemáticos y con alumnos de Educación Infantil. Estos autores observaron que la utilización de la música produjo un impacto positivo en el aprendizaje, logrando que se alcanzaran los conocimientos básicos de las Matemáticas en un ambiente agradable, fuertemente motivado tanto en la dimensión física como en la emocional. 
Así pues, es conveniente iniciar a los alumnos en el mundo de las matemáticas de un modo más lúdico y relajado a través de actividades prácticas (Noll, 2014), pues los prepararán para la adquisición de las competencias matemáticas dentro de un marco afectivo.

Además, como señala Canals (2001), en la etapa infantil los contenidos matemáticos no se deben enseñar aislados sino globalizado con la educación sensorial y del lenguaje; con la psicomotricidad, la plástica, los cuentos, el conocimiento del medio y por supuesto la música.

Así mismo, los estudios de Mato y Muñoz (2010) corroboran la hipótesis ya conocida de que el interés por las matemáticas va progresivamente disminuyendo conforme aumenta el curso de estudio, por lo que los primeros años son fundamentales para la adquisición del desarrollo del pensamiento matemático sobre todo en la prevención y corrección de actitudes negativas hacía la materia.

Por otra parte, nuestra investigación nos ha llevado a redescubrir textos compuestos fundamentalmente por canciones muy lúdicas y olvidadas en la enseñanza de las matemáticas (Sanuy y Cortés, 1978; Hernández, 1984), así como otros más actuales que incluyen canciones de contenidos matemáticos adecuadas para los estudiantes de Infantil y Primaria como: Aznárez (1992); San Andrés (2000) y, Bernal, Calvo y Martín (2000). Internet también ha sido una potente fuente que nos ha proporcionado canciones variadas e idóneas para nuestro cometido que nos muestran el poder de la música como transmisora de conocimientos. Al final de este artículo se incluye una webgrafía que puede ser revisada incluso para ampliar las canciones a otros ámbitos.

\section{OBJETIVO Y METODOLOGÍA DE LA INVESTIGACIÓN}

El objetivo de este trabajo es obtener una guía de canciones de contenidos relacionados con la enseñanza y el aprendizaje de las matemáticas en la etapa de Educación Infantil y Primaria (primer y segundo curso) para que pueda ser utilizada por los docentes que imparten estas enseñanzas.

En un primer momento se realizó un análisis del currículo de la Comunidad de Extremadura, de lo que se extrajeron los diferentes contenidos matemáticos que se imparten en los cursos de estudio.

A partir del currículo, se comenzó la búsqueda de canciones en diferentes textos y artículos de las bibliotecas, así como en distintas páginas web, pues Internet sirvió como principal fuente para la obtención de canciones de contenidos matemáticos, debido a la amplia cantidad de información que podemos encontrar en la red.

Tras una primera revisión, se obtuvieron 238 canciones diferentes sobre contenidos matemáticos diversos, de las que se seleccionaron 98 tras aplicar los siguientes criterios:

- Presencia implícita o explícita de contenidos matemáticos en la canción.

- Adecuación de las canciones para alguna de las etapas educativas a las que se destina nuestra investigación, o incluso para ambas.

- Existencia de partitura o acordes para guitarra, para que ésta pueda ser trabajada en el aula y sean útiles para los docentes que imparten estas enseñanzas

No se han excluido las canciones encontradas en inglés que trabajan contenidos matemáticos de las mencionadas etapas, ya que en ambas se imparte la lengua inglesa y consideramos de especial importancia la adquisición de vocabulario y contenidos matemáticos en otra lengua. 
Para el análisis y la clasificación de las canciones de contenidos matemáticos se han establecido unas categorías determinadas. El instrumento de análisis lo constituyen tales categorías junto con los elementos que se necesitan para estudiar cada una de las mismas; como la descripción, los criterios, y las tablas y gráficos para la recogida y exposición de datos, entre otros.

La formación de categorías de análisis no es un proceso sencillo ya que está sujeto a constantes modificaciones. Durante los procesos de análisis de las canciones tales categorías han sufrido cambios, puesto que transforman aspectos descriptivos de las mismas, se añaden o se eliminan y además se perfecciona el tratamiento y análisis de las canciones dentro de cada categoría.

En la Tabla 1 se presentan las categorías definitivas del estudio que han resultado del proceso expuesto y su código de identificación.

Tabla 1. Categorías de Análisis.

\begin{tabular}{cc}
\hline CATEGORÍA & CÓDIGO \\
\hline CONJUNTOS & $\mathrm{C}_{\circ}$ \\
CONCEPTOS PRENUMÉRICOS & $\mathrm{C}_{\mathrm{P}}$ \\
NÚMEROS & $\mathrm{N}$ \\
OPERACIONES Y RESOLUCIÓN DE PROBLEMAS & $\mathrm{O}_{\mathrm{RP}}$ \\
GEOMETRÍA & $\mathrm{G}$ \\
MEDIDA & $\mathrm{M}$ \\
ORIENTACIÓN ESPACIAL & $\mathrm{O}_{\mathrm{E}}$ \\
\hline
\end{tabular}

Todas las categorías están relacionadas con la etapa de Educación Infantil. Las categorías Conceptos prenuméricos y de Conjuntos tratan los contenidos previos a la adquisición del número y las categorías de Números, Operaciones y Resolución de problemas trabajan la construcción de series numéricas y la utilización de los números para contar mediante juegos y actividades sencillas. Igualmente, las canciones que aparecen insertas en la categoría de Orientación Espacial hablan de la posición que ocupan los objetos en el espacio y la situación de los objetos con relación a uno mismo. No menos importantes son las categorías de Geometría y Medida en las que los alumnos se inician en el conocimiento de los atributos de los objetos como su forma, y en la estimación y observación de distintas medidas.

Con respecto a la Educación Primaria, estas categorías están relacionadas con los bloques del currículo, concretamente con Resolución de problemas, Números y, Geometría y Medida. No se han incluido el bloque Estadística y Probabilidad pues no se han encontrado canciones relacionadas con este tema. Solamente Lesser $(2000,2001)$ y Robertson y Lesser (2013) utiliza la música como una herramienta de análisis de datos, exploración de problemas y estadísticas relacionadas con las canciones principalmente en Secundaria. Propone una amplia variedad de datos cuantitativos que se pueden recopilar y resumir, como la longitud del tiempo de las canciones o la cantidad de palabras en los títulos de las canciones más de moda. Recomiendan la representación de estos datos mediante gráficos como un histograma o un diagrama de caja y bigotes. Aunque esta serie de actividades también se pueden hacer en Primaria, como dicen los autores, no se corresponden con el planteamiento de nuestra investigación. 
A su vez, cada canción se enmarca en una categoría. Si puede ser asignada a dos categorías se elegirá la categoría en función del contenido que se trabaje de forma mayoritaria en la canción, y se detallará en el apartado observaciones de su ficha la relación de la canción con los contenidos propios de otra categoría.

Por otra parte, la codificación de las canciones se ha realizado teniendo en cuenta tres elementos: enumeración ordinal (de 1 a 98), curso y etapa educativa (ver Tabla 2) y categoría a la que pertenece (ver Tabla 1).

Tabla 2. Codificación de los Cursos y Etapas Educativas.

\begin{tabular}{ccc}
\hline ETAPA EDUCATIVA & CURSO & CÓDIGO \\
\hline \multirow{2}{*}{ Educación Infantil } & 3 años & 31 \\
& 4 años & 41 \\
& 5 años & 51 \\
\hline \multirow{2}{*}{ Educación Primaria } & Primer curso & $1 \mathrm{P}$ \\
& Segundo curso & $2 \mathrm{P}$ \\
\hline
\end{tabular}

La ficha genérica (Figura 1) sirve de modelo para presentar cada una de las canciones seleccionadas con todos los detalles que definen y caracterizan una canción, y la categoría a la que pertenece.

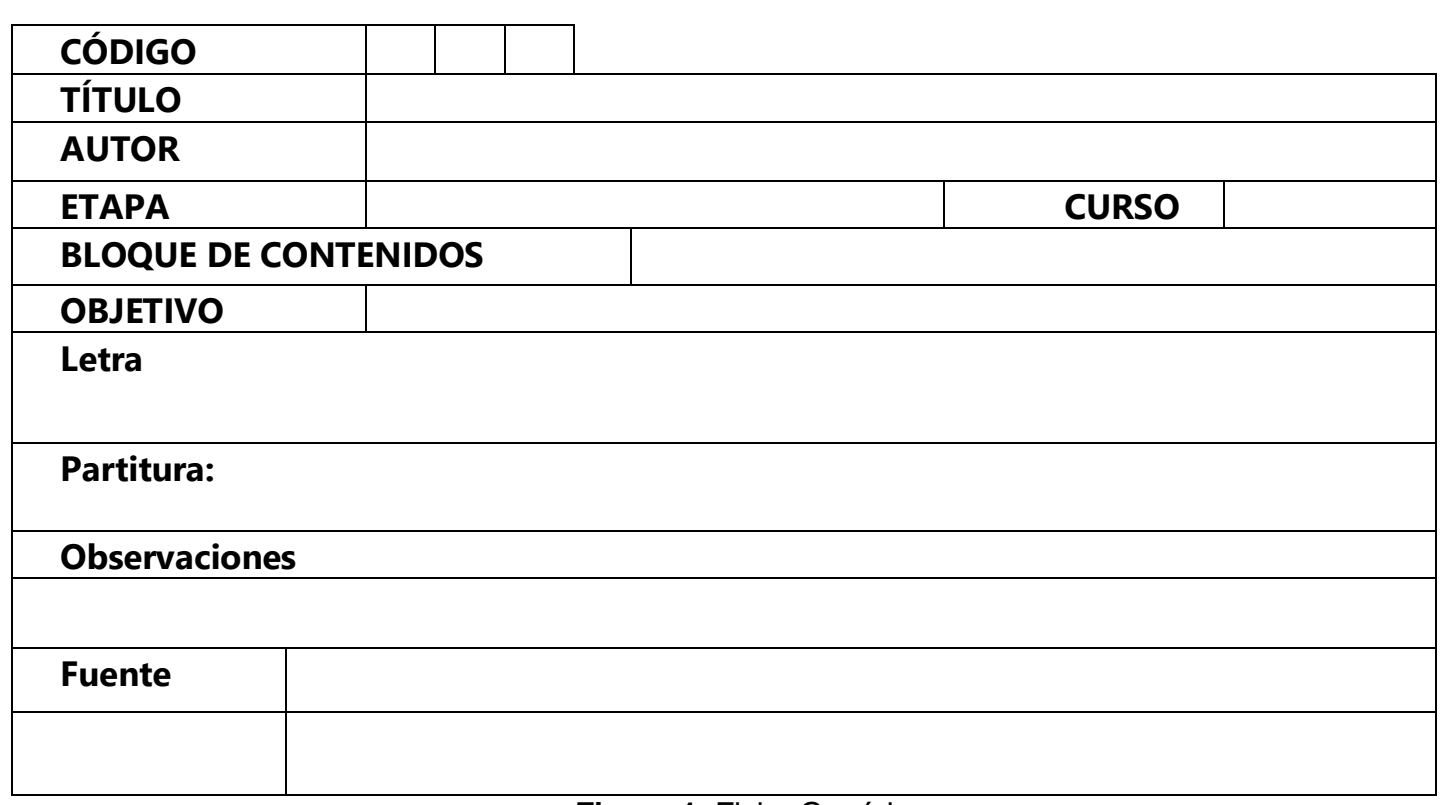

Figura 1. Ficha Genérica.

Ésta consta de 11 secciones que son:

1. Código: Explicado anteriormente.

2. Título: Nombre de la canción que se presenta en la ficha, que ha sido respetado sin sufrir modificaciones, y por tanto aparece igual que en el libro o web de la que ha sido extraída.

3. Autor: De la letra de la canción y de la música en caso de que éste fuera distinto. Además, se presenta el año de creación de la canción, si lo hubiera.

4. Etapa: Es la etapa educativa a la que va dirigida esta canción (Educación Infantil o/y Educación Primaria).

5. Curso: Al que va dirigida la canción. Tal apartado se encuentra segmentado en el caso de que la canción vaya destinada a las dos etapas educativas a las que se dirige esta investigación, así como a distintos años o cursos. 
6. Bloque de contenidos: Aparece la categoría de análisis a la que pertenece la canción. Asimismo, en algunas canciones se ha hecho una subclasificación atendiendo al contenido específico que se trabaja en ella; por ejemplo, en la categoría de medida, se especifica si es medida de longitud, de peso o del tiempo.

7. Objetivo: Conceptos o procedimientos que se pretende y se quiere que los alumnos aprendan o refuercen con esa canción.

8. Letra: Original de la canción.

9. Partitura: Texto escrito donde se anotan los sonidos que han de ejecutarse.

10. Observaciones: Se trata de sugerencias con relación a cómo abordar la canción a la hora de cantarla en el aula como, por ejemplo, bailes y gestos que se le puede añadir, o materiales adecuados para acompañarla. También se añaden sugerencias para ampliar la canción sin necesidad de que su melodía se vea afectada. Del mismo modo en algunos casos encontramos en esta sección actividades que se han elaborado para acompañar las canciones, y así conseguir los objetivos que con ella se pretenden.

11. Fuente: Referencia bibliográfica o página web de la que ha sido extraída la letra y partitura de la canción.

\section{DATOS Y RESULTADOS}

En total se han obtenido 98 canciones relacionadas con contenidos matemáticos de El y de EP haciendo uso de fuentes bibliográficas y gran variedad de páginas webs. De estas canciones, se ha realizado una clasificación en base a las categorías establecidas, como se muestra en el siguiente gráfico (Figura 2) en el que aparecen las categorías, nombradas con su código correspondiente, y el número de canciones incluidas en ellas.

La tendencia de trabajar el bloque de los Números en la escuela un mayor tiempo en detrimento de los otros bloques se observa en este gráfico donde la categoría Números (N) sobresale sobre todas las demás. Este resultado se reafirma si además añadimos las canciones de la categoría Operaciones y Resolución de problemas $\left(\mathrm{O}_{\mathrm{RP}}\right)$, que está también relacionada con los números.

Para la presentación de las canciones se han elaborado tablas, una para cada categoría, y en cada una de ellas aparece el nombre y el código que corresponde a cada canción. Vamos a realizar una breve descripción de forma genérica de los contenidos matemáticos que encontramos en las canciones pertenecientes a cada categoría.

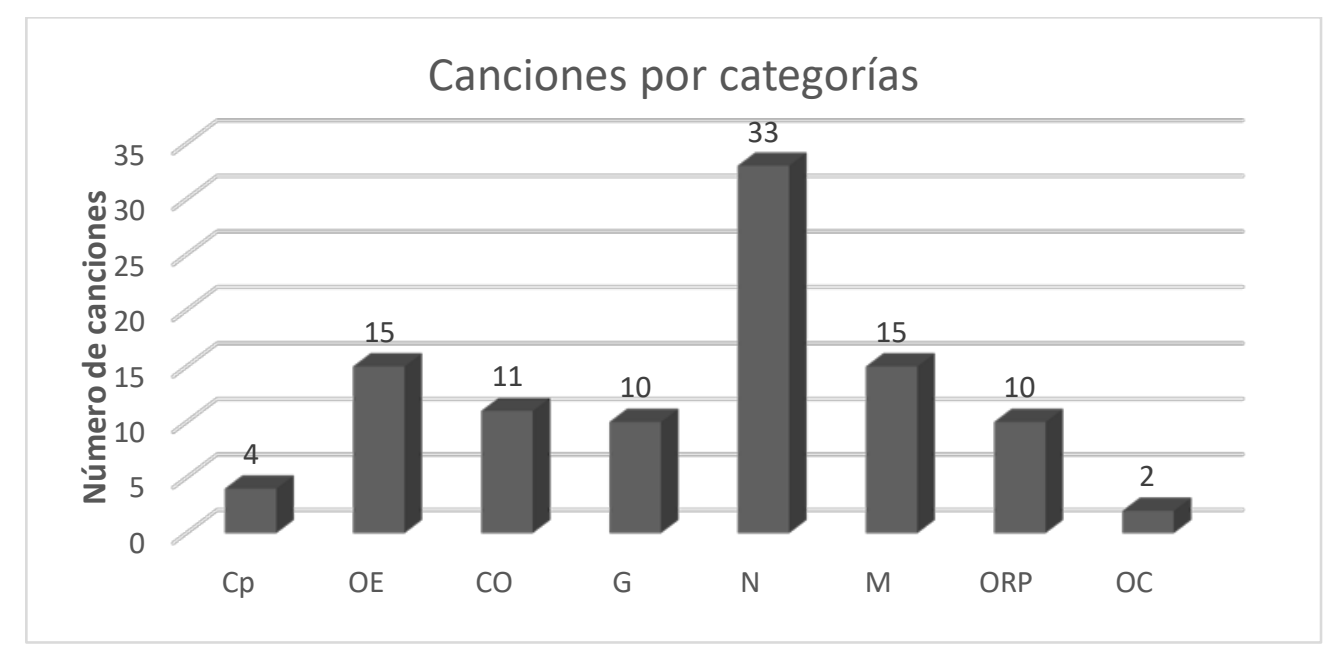

Figura 2. Gráfica Comparativa de Canciones por Categorías de Análisis. 
Categoría Conjuntos (Co). Se incluyen 11 canciones y a través de éstas, se refuerzan el concepto de pertenencia y no pertenencia a un conjunto, así como la siembra de la clasificación, concepto muy importante para la adquisición del concepto de número. La Tabla 3 muestra la relación de canciones de esta categoría.

Tabla 3. Categoría de Conjuntos.

\begin{tabular}{llll}
\hline \multicolumn{2}{c}{ Código } & \multicolumn{1}{c}{ CONJUNTOS } \\
\hline $\mathbf{2 0}$ & Co & Nombre de la canción \\
\hline $\mathbf{2 1}$ & Co & Colecciono mariposas \\
$\mathbf{2 2}$ & Co & Colores \\
$\mathbf{2 3}$ & Co & Co & Colores y colores \\
$\mathbf{2 4}$ & Co & Colores primarios \\
$\mathbf{2 5}$ & I & Co & Colores primarios y secundarios \\
$\mathbf{2 6}$ & I & Co & Tres colores \\
$\mathbf{2 7}$ & I & Co & Juguemos a los colores \\
$\mathbf{2 8}$ & Co & Señalar un color \\
$\mathbf{2 9}$ & Co & Baile de colores \\
$\mathbf{3 0}$ & Co & Co & Verde esperanza \\
\hline
\end{tabular}

Categoría Conceptos Prenuméricos (Cp). Estas canciones refuerzan los conceptos prenuméricos de correspondencia y seriación, que junto con la conservación de la cantidad y la inclusión de las partes en el todo, predisponen a los alumnos para una buena enseñanza del número. La Tabla 4 muestra la relación de canciones de esta categoría.

Tabla 4. Categoría Conceptos Prenuméricos.

\begin{tabular}{lllll}
\hline & & & CONCEPTOS PRENUMÉRICOS \\
\hline \multicolumn{3}{c}{ Código } & \multicolumn{1}{c}{ Nombre de la canción } \\
\hline $\mathbf{1}$ & 3I & $\mathbf{4}$ & CP & Los animales. \\
$\mathbf{2}$ & $4 I$ & 51 & CP & Tamaños. \\
$\mathbf{3}$ & I & & CP & Más largos que un ciempiés. \\
$\mathbf{4}$ & I & & CP & He visto un árbol muy alto. \\
\hline
\end{tabular}

Categoría Números (N). Es la categoría con mayor número de canciones (33), las cuales se muestran en la Tabla 5. Éstas ayudan al alumno a aprender y reforzar el estudio del número como cardinal, así como para trabajar el orden correcto de la sucesión numérica creciente y decreciente. Una característica importante es que muchas de las letras de estas canciones, pueden modificarse y ampliarse para seguir contando hasta el número que se considere oportuno o bien para realizar la misma actividad con otros números. Las canciones relacionan los números con conjuntos de la vida ordinaria y algunas son en inglés para reforzar el aprendizaje de los primeros números en otra lengua. 
Tabla 5. Categoría Números.

\begin{tabular}{|c|c|c|c|c|c|c|c|}
\hline \multirow{2}{*}{\multicolumn{3}{|c|}{ Código }} & \multicolumn{5}{|c|}{ CONJUNTOS } \\
\hline & & & Nombre de la canción & & ódig & & Nombre de la canción \\
\hline 4 & & & & 5 & & & \\
\hline $\begin{array}{l}1 \\
4\end{array}$ & 31 & $\mathrm{~N}$ & Dos perritos & $\begin{array}{l}8 \\
5\end{array}$ & I & $\mathrm{N}$ & A contar ya \\
\hline 2 & 31 & $\mathrm{~N}$ & Mi barba tiene tres pelos & 9 & I & $\mathrm{N}$ & Te enseño a contar \\
\hline 4 & & & & 6 & I & $\mathrm{N}$ & One, two, three, four, five, once I caught a \\
\hline $\begin{array}{l}3 \\
4\end{array}$ & 31 & $\mathrm{~N}$ & Cinco lobitos & $\begin{array}{l}0 \\
6\end{array}$ & & & fish alive \\
\hline $\begin{array}{l}4 \\
4\end{array}$ & 31 & $\mathrm{~N}$ & Juego de las baldosas & $\begin{array}{l}1 \\
6\end{array}$ & I & $\mathrm{N}$ & Yo tenía diez perritos \\
\hline $\begin{array}{l}5 \\
4\end{array}$ & $\begin{array}{l}31 \\
31\end{array}$ & $\mathrm{~N}$ & Un, dos, tres & $\begin{array}{l}2 \\
6\end{array}$ & I & $\mathrm{N}$ & Un ratoncito sale de la cueva \\
\hline $\begin{array}{l}6 \\
4\end{array}$ & $\begin{array}{l}41 \\
31\end{array}$ & $\mathrm{~N}$ & El cajón & $\begin{array}{l}3 \\
6\end{array}$ & I & $\mathrm{N}$ & Un elefante \\
\hline $\begin{array}{l}7 \\
4\end{array}$ & $\begin{array}{l}41 \\
31\end{array}$ & $\mathrm{~N}$ & Canción de los números & $\begin{array}{l}4 \\
6\end{array}$ & I & $\mathrm{N}$ & Yo primero \\
\hline 8 & 41 & $\mathrm{~N}$ & Fivelittle monkeys & 5 & I & $\mathrm{N}$ & Cuento y cuento \\
\hline 4 & 31 & & Five little ducks went swimming & 6 & & & \\
\hline 9 & 41 & $N$ & one day & 6 & IIP & $\mathrm{N}$ & Ten in thebed \\
\hline 5 & 3I & & & 6 & 511 & & \\
\hline $\begin{array}{l}0 \\
5\end{array}$ & 41 & $\mathrm{~N}$ & La gallina & $\begin{array}{l}7 \\
6\end{array}$ & $\begin{array}{l}P \\
5 I 1\end{array}$ & $\mathrm{~N}$ & Epidemia en el colegio \\
\hline $\begin{array}{l}1 \\
5\end{array}$ & I & $\mathrm{N}$ & De codín, de codán & $\begin{array}{l}8 \\
6\end{array}$ & $\mathrm{P}$ & $\mathrm{N}$ & Contar hasta veinte \\
\hline $\begin{array}{l}2 \\
5\end{array}$ & I & $\mathrm{N}$ & Uno, una & $\begin{array}{l}9 \\
7\end{array}$ & $\mathrm{P}$ & $\mathrm{N}$ & Contar hasta treinta \\
\hline $\begin{array}{l}3 \\
5\end{array}$ & I & $\mathrm{N}$ & La gallina Ponicaca & $\begin{array}{l}0 \\
7\end{array}$ & $\mathrm{P}$ & $\mathrm{N}$ & Treinta y dos \\
\hline $\begin{array}{l}4 \\
5\end{array}$ & I & $\mathrm{N}$ & La gallina Turuleca & $\begin{array}{l}1 \\
7\end{array}$ & $\mathrm{P}$ & $\mathrm{N}$ & Contar hasta cuarenta \\
\hline $\begin{array}{l}5 \\
5\end{array}$ & I & $\mathrm{N}$ & Un, dos, tres & $\begin{array}{l}2 \\
7\end{array}$ & $\mathrm{P}$ & $\mathrm{N}$ & Uno a cuarenta \\
\hline $\begin{array}{l}6 \\
5\end{array}$ & I & $\mathrm{N}$ & Aprender a contar & 3 & $\mathrm{P}$ & $\mathrm{N}$ & The twelve days of Christmas \\
\hline 7 & I & $\mathrm{N}$ & Contar hasta diez & & & & \\
\hline
\end{tabular}

Categoría Operaciones y Resolución de Problemas $\left(O_{R P}\right)$. Se trata de canciones para iniciar a los alumnos en el aprendizaje de las operaciones y fortalecer su mecánica. Varias de las canciones tratan situaciones problemáticas en las que los alumnos tienen que utilizar la suma o la resta para resolverlas de forma oral, a la vez que cantan la canción, desarrollando el cálculo mental y la agilidad en la automatización de las operaciones.

Tabla 6. Categoría Operaciones y Resolución de Problemas.

\begin{tabular}{|c|c|c|c|c|}
\hline \multicolumn{5}{|c|}{ OPERACIONES Y RESOLUCIÓN DE PROBLEMAS } \\
\hline \multicolumn{4}{|c|}{ Código } & Nombre de la canción \\
\hline 89 & 51 & $\mathrm{P}$ & $\mathrm{O}_{\mathrm{RP}}$ & Sumar hasta tres \\
\hline 90 & 51 & $\mathrm{P}$ & $\mathrm{O}_{\mathrm{RP}}$ & A sumar números iguales \\
\hline 91 & 51 & $\mathrm{P}$ & $\mathrm{O}_{\mathrm{RP}}$ & Brinca la tablita \\
\hline 92 & 51 & $P$ & $\mathrm{O}_{\mathrm{RP}}$ & Manzanas \\
\hline 93 & 51 & $P$ & $\mathrm{O}_{\mathrm{RP}}$ & ¿Cuántos años cumplirás? \\
\hline 94 & 51 & $\mathrm{P}$ & $\mathrm{O}_{\mathrm{RP}}$ & Una hormiguita \\
\hline 95 & 51 & $P$ & $\mathrm{O}_{\mathrm{RP}}$ & Hoy vamos a restar \\
\hline 96 & 51 & $P$ & $\mathrm{O}_{\mathrm{RP}}$ & Diez patos \\
\hline 97 & \multicolumn{2}{|c|}{$P$} & $\mathrm{O}_{\mathrm{RP}}$ & Soy el farolero \\
\hline 98 & \multicolumn{2}{|c|}{$\mathrm{P}$} & $\mathrm{O}_{\mathrm{RP}}$ & Sumar y restar \\
\hline
\end{tabular}


Categoría Geometría (G). En esta categoría se trabajan las figuras geométricas básicas como círculo, cuadrado, triángulo y rectángulo, así como canciones específicas para conocer las propiedades de estas, por ejemplo, de los triángulos escaleno, isósceles y equilátero, y aprender a diferenciarlos.

Tabla 7. Categoría Geometría.

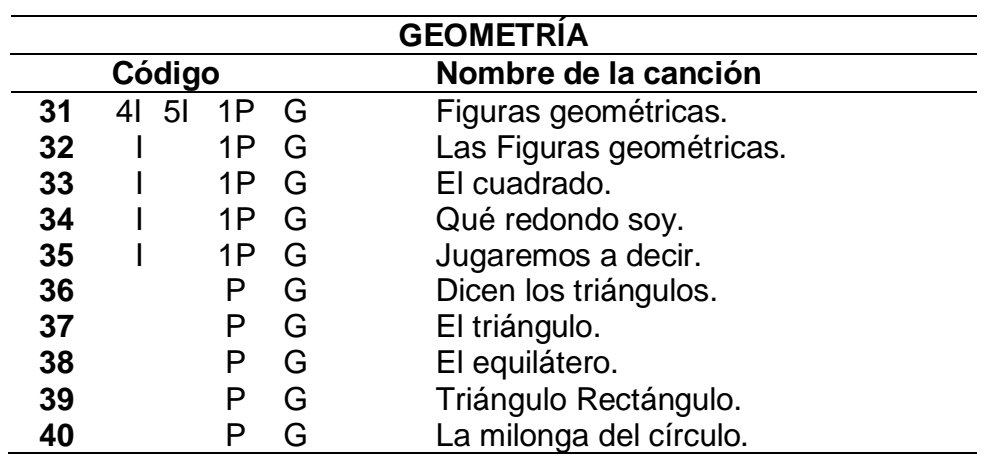

También, en estas canciones se establecen relaciones entre las formas geométricas en los objetos del entorno para conseguir el objetivo del currículo de relacionar la geometría con la vida ordinaria del alumno. En esta categoría hemos encontrado 10 canciones como puede observarse en la Tabla 7.

Categoría Medida (M). Hay que señalar que en algunas letras pueden modificarse los números de las operaciones sin necesidad de que su melodía se vea alterada, por ejemplo, la 92, 93, 94 y 96. La relación de canciones está contenida en la Tabla 6.

La categoría de medida incluye canciones genéricas en las que se exponen unidades de medida no convencionales como el paso, el palmo y el codo; y unidades de medidas convencionales del Sistema Internacional como el metro, el litro y el kilo. Se pretende con ello que los alumnos comprendan tales conceptos según la magnitud correspondiente. En la magnitud tiempo se trabajan las horas, los minutos y segundos, los días de la semana, las estaciones y los meses del año; en la magnitud masa, el concepto de kilo y que aprenda que las masas iguales no varían en función de la materia, y en la medida de longitud se trabajan conceptos básicos como el centímetro, el metro y el kilómetro.

Como podemos observar, en esta muestra de 15 canciones, se abordan las magnitudes que se enseñan en Infantil y Primaria (Tabla 8).

Tabla 8. Categoría Medida.

\begin{tabular}{|c|c|c|c|c|}
\hline \multicolumn{5}{|c|}{ LA MEDIDA } \\
\hline \multicolumn{4}{|c|}{ Código } & Nombre de la canción \\
\hline 74 & 1 & & $M$ & Siete días \\
\hline 75 & I & & M & Días de la semana \\
\hline 76 & 41 & $51 \mathrm{P}$ & $M$ & Adivina la estación \\
\hline 77 & 41 & $51 \mathrm{P}$ & M & Paso a paso \\
\hline 78 & 51 & $P$ & M & Los esqueletos \\
\hline 79 & 51 & $P$ & M & Las horas \\
\hline 80 & 51 & $P$ & M & Las estaciones \\
\hline 81 & 51 & $P$ & $M$ & Meses y estaciones \\
\hline 82 & 1 & $\mathrm{P}$ & $M$ & Doce meses \\
\hline 83 & l & $P$ & $M$ & Canto de los meses \\
\hline 84 & i & $P$ & $\mathrm{M}$ & Estaciones y meses \\
\hline 85 & $P$ & & $M$ & Horas, minutos y segundos \\
\hline 86 & $P$ & & $M$ & Peso dos cosas \\
\hline 87 & $\mathrm{P}$ & & M & El Kilo \\
\hline 88 & $\mathrm{P}$ & & M & Kilómetro \\
\hline
\end{tabular}


Categoría Orientación Espacial $\left(\boldsymbol{O}_{\mathrm{E}}\right)$. Es imprescindible que el niño sepa situarse en el espacio con relación a sí mismo y en relación con los objetos antes de iniciarlo en nociones de tipo geométrico (Hernández, 1984). Y ese es el objetivo de las canciones incluidas en esta categoría de Orientación Espacial.

Tabla 9. Categoría Orientación espacial.

\begin{tabular}{ccll}
\hline \multicolumn{4}{c}{ ORIENTACIÓN ESPACIAL } \\
\hline $\mathbf{5}$ & Código & \multicolumn{1}{c}{ Nombre de la canción } \\
\hline $\mathbf{6}$ & $\mathrm{O}_{\mathrm{E}}$ & $\mathrm{O}_{\mathrm{E}}$ & Mirad, mirad allí. \\
$\mathbf{7}$ & $\mathrm{O} \mathrm{O}_{\mathrm{E}}$ & Mi ladorda y derecha. \\
$\mathbf{8}$ & $\mathrm{O}$ & Mi lado izquierdo. \\
$\mathbf{9}$ & $\mathrm{O}$ & $\mathrm{O}_{\mathrm{E}}$ & Arriba y abajo. \\
$\mathbf{1 0}$ & $\mathrm{O}$ & A la zapatilla por detrás. \\
$\mathbf{1 1}$ & $\mathrm{O}$ & $\mathrm{O}_{\mathrm{E}}$ & Las gafas de mi tío. \\
$\mathbf{1 2}$ & $\mathrm{O}$ & $\mathrm{O}_{\mathrm{E}}$ & Jugando con las piedras. \\
$\mathbf{1 3}$ & $\mathrm{O}$ & ¿Dónde está la mosca? \\
$\mathbf{1 4}$ & $\mathrm{O}$ & $\mathrm{O}_{\mathrm{E}}$ & Fuera y dentro. \\
$\mathbf{1 5}$ & $\mathrm{O}$ & $\mathrm{O}_{\mathrm{E}}$ & Juego del objeto perdido. \\
$\mathbf{1 6}$ & $\mathrm{O}$ & Los exploradores. \\
$\mathbf{1 7}$ & $\mathrm{O}$ & $\mathrm{O}_{\mathrm{E}}$ & La rana. \\
$\mathbf{1 8}$ & $\mathrm{O}$ & $\mathrm{O}_{\mathrm{E}}$ & En casa y fuera de casa. \\
$\mathbf{1 9}$ & $\mathrm{I}$ & $\mathrm{O}_{\mathrm{E}}$ & Entrar y salir. \\
\hline
\end{tabular}

Se trata de 15 canciones que tocan las distintas etapas de la orientación espacial como son: la orientación en su propio cuerpo y en su entorno próximo; así como la localización de objetos en el espacio atendiendo a la clasificación que se hace en autor y autor (2017).

Las canciones (Tabla 9) están orientadas a trabajar la lateralidad de los alumnos, así como otras nociones básicas de orientación como arriba y abajo, encima y debajo, delante y detrás, etc. Resulta interesante resaltar que la mayoría de las canciones son muy dinámicas y dan pie a realizar juegos, dramatizaciones, y diversas actividades motivadoras.

Todas las canciones seleccionadas pueden ser consultadas en la página web Guía de canciones matemáticas para Educación Infantil y Primaria en la siguiente dirección:

\section{http://guiadecancionesmatematicas.piojosoft.es/}

La página web cuenta con 5 secciones: inicio, contacto, ficha genérica,codificación de ficha y canciones, siendo esta última la sección más interesante en la cual se despliega un menú con varios enlaces que corresponden con las categorías. Pulsando la categoría deseada accederemos a las canciones de esa categoría, primero al listado general y luego las fichas, que pueden descargarse. 


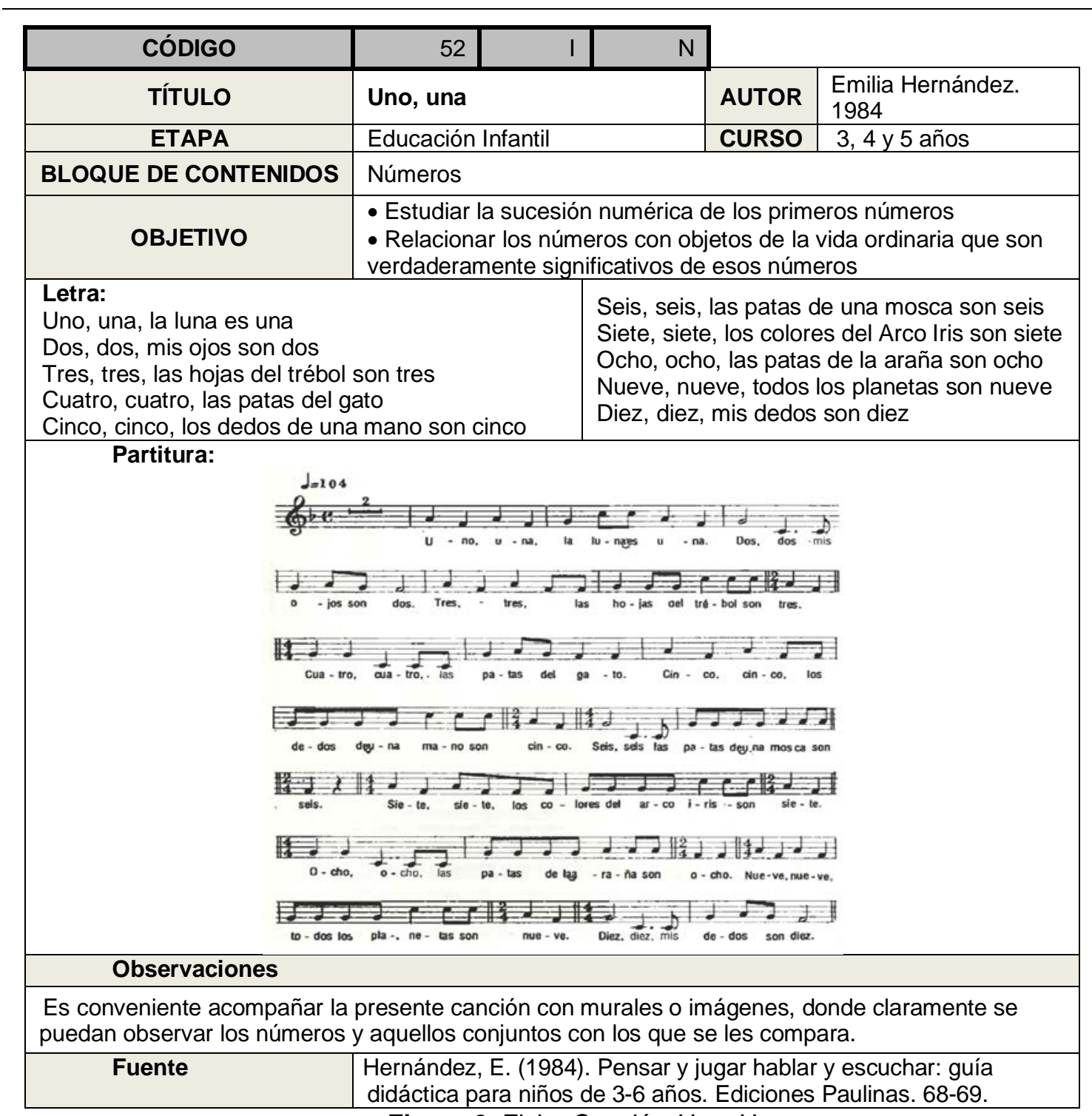

Figura 3. Ficha Canción Uno, Una.

\section{Dos ejemplos de cómo utilizar las canciones en el aula.}

Mostramos dos ejemplos de las canciones con contenidos matemáticos relacionados con el currículo de El y/o EP. Exponemos su correspondiente ficha comentada, así como varias actividades matemáticas para trabajar los contenidos de las canciones. Las canciones pertenecen a las categorías de Números $(\mathrm{N})$, y Operaciones y Resolución de Problemas $\left(\mathrm{O}_{\mathrm{RP}}\right)$.

\section{1- Canción Uno, Una.}

La primera es la canción Uno, Una (Figura 3) que es una buena alternativa a la canción con código (47, 3 y 4l, N), la ya conocida Canción de los números en la que se cosifican los números (El uno es un soldado haciendo la instrucción, El dos es un patito que está tomando el sol...). Sin embargo, en Uno, Una se compara el número uno con un objeto de la vida ordinaria, en este caso la Luna, que además es un objeto que se caracteriza porque es muy representativo de dicho número pues solo hay una. De igual manera se asocia el número dos con los ojos de la cara, que igualmente solo hay dos y no se cosifica con un patito como se hace en la Canción de los números. En general en la canción se buscan objetos de la vida ordinaria muy representativos del número correspondiente. 
Se pueden realizar actividades mientras los niños cantan la canción. Por ejemplo, distribuiremos por la clase los aros y en cada uno de ellos depositaremos una tarjeta en la que aparezca un número del 1 al 10 y los objetos que se mencionan en la canción para cada número.

Se comenzará a cantar la canción Uno, Una realizando una pausa al finalizar cada oración. El maestro señalará un alumno cuando se mencione el número en la canción, y éste tendrá que buscar el aro que contenga tal número y una vez que lo encuentre coger la tarjeta y terminar la frase. Por ejemplo: la docente dice: Uno, Una y el alumno cuando encuentra el número termina: La Luna es una. Tras encontrar el aro correspondiente tendrá que depositar dentro del aro la cantidad de cosas que corresponda con ese número y tendrá que quedarse dentro del aro. Realizaremos la misma acción con el resto de alumnos hasta finalizar la canción. Después podemos reforzar con preguntas como: ¿dónde está el número 4?,¿cuántos objetos hay dentro del círculo? Cada objeto que haya en el círculo será contado entre todos los alumnos de la clase en voz alta.

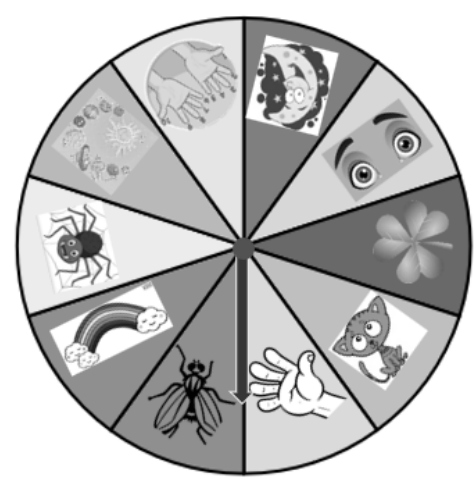

Figura 4. Rueda ilustrada empleada para trabajar la canción Uno, Una.

En otra actividad llamada La rueda de los números fabricaremos un círculo que contará con 10 sectores (Figura 4) y en cada uno estarán los dibujos que representan los objetos mencionados en la canción, los cuales representan los números del 1 al 10. Tendremos también 10 pinzas que llevan escrito el símbolo numérico correspondiente.

De forma individual, seleccionaremos a un alumno al azar que hará girar la rueda. Cuando la ruleta pare, el alumno se fija en la sección que ha quedado en la flecha y tendrá que decir qué ve en la imagen, contar los elementos y posteriormente colocar en esa sección la pinza que tenga escrito el número que corresponde. Posteriormente, girará la rueda otro alumno y así sucesivamente mientras se canta la canción.

\section{2- Canción ¿Cuántos años cumplirás?}

Esta canción (Figura 5) pertenece a la categoría Operaciones y Resolución de Problemas y se puede modificar fácilmente, como se menciona en observaciones. Tan solo es necesario cambiar los números que se quieren descomponer y la descomposición del mismo. Además, podemos alargar la canción tanto como queramos, añadiendo la descomposición de más números y no solo de dos, lo que es muy adecuado para aumentar su complejidad de forma progresiva.

Para trabajar en mayor medida la iniciación a la suma y la descomposición de números, sería conveniente que la canción se acompañara con actividades, tales como las que se proponen a continuación. 
Se puede cantar la canción mientras con los dedos de las dos manos se va escenificando la descomposición. También, pegar y colorear velas en una ficha como la que se muestra en la Figura 6. Cada alumno tendrá que decirnos las tres maneras diferentes en las que se puede descomponer su edad, en función de la distribución de las velas en cada tarta.

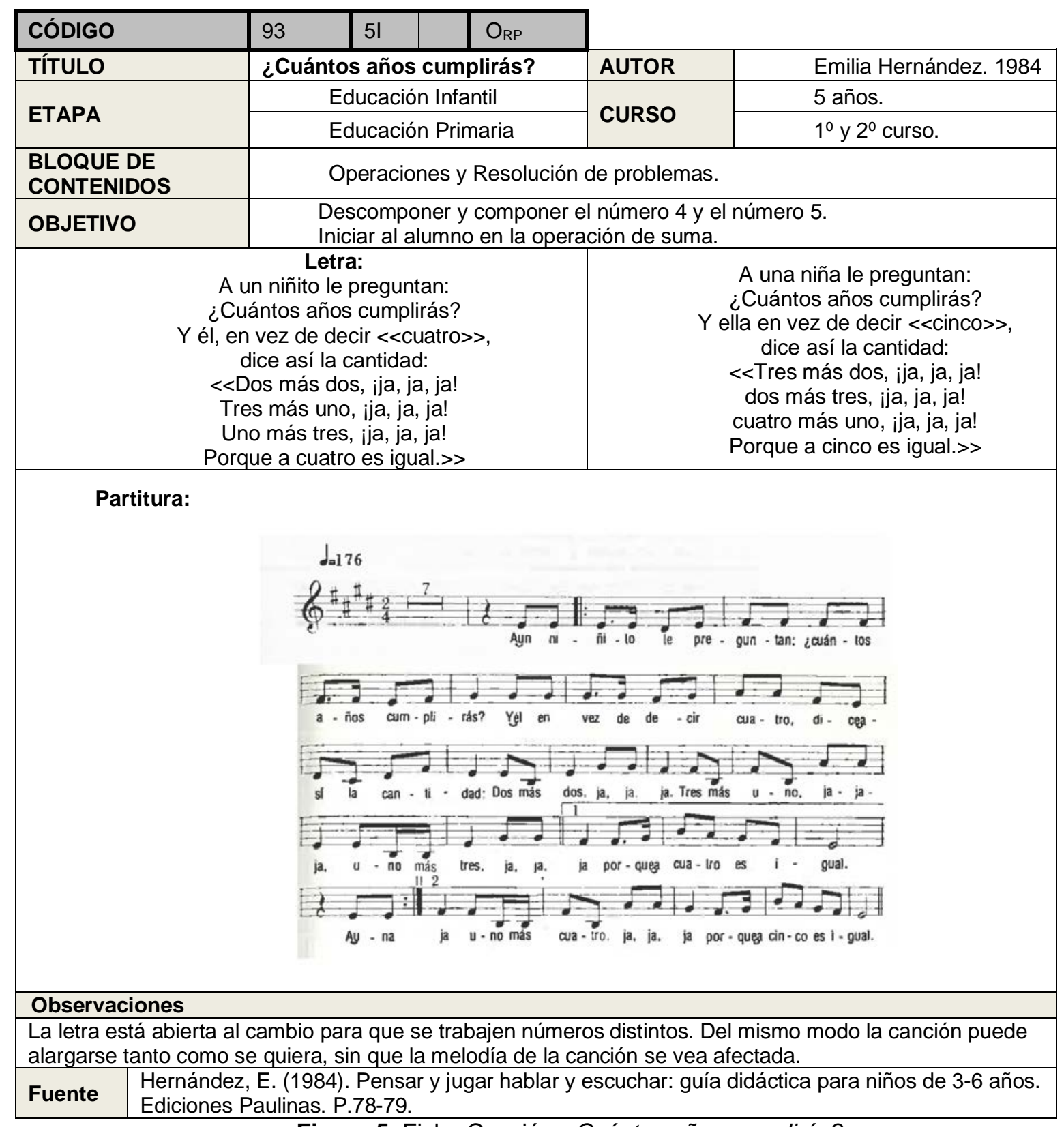

Figura 5. Ficha Canción ¿Cuántos años cumplirás? 
MI TARTA DE CUMPLEAÑOS.

FECHA:

Recorta las velas que corresponden con tu edad, y colócalas en las tartas de diferente manera. Colorea las velas de cada tarta de dos colores.
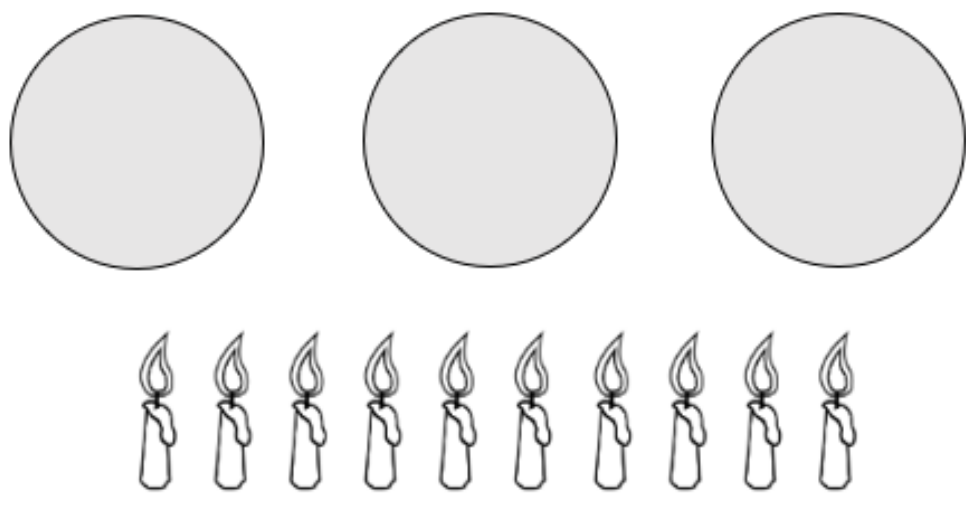

Figura 6. Ficha Mi tarta de cumpleaños.

Por último, una actividad interesante es la llamada Cada coche en su lugar. Para jugar solo necesitaremos fichas de dominó y un tablero para colocar las fichas. Unos modelos de tablero pueden ser el que se presenta en la Figura 7.

Se trata de jugar con las fichas de dominó como si fueran coches y el tablero, un parking. En la actividad propuesta en la Figura 7-1, se le repartirá a cada alumno 5 fichas y ganará el primero que se quede sin fichas o bien cuando completen el tablero. Cada ficha será un coche y tendrán que aparcarlo en su parada correspondiente que es el número resultante de la suma de los puntos de la ficha de dominó. Si en un turno el alumno no puede colocar ninguna ficha, tendrá que robar. Otra opción es la mostrada en la Figura 7-2, en este caso el juego se realiza con un tablero de un solo número y el alumno debe realizar, jugando, varias descomposiciones del mismo número.

Estos ejemplos nos muestran cómo pueden integrarse las canciones dentro del contexto del contenido y las actividades que se están trabajando en ese momento. Nuestro objetivo es que la canción, no sea un mero entretenimiento lúdico, sino que sea un recurso de apoyo a la enseñanza en Educación Infantil y Primaria.
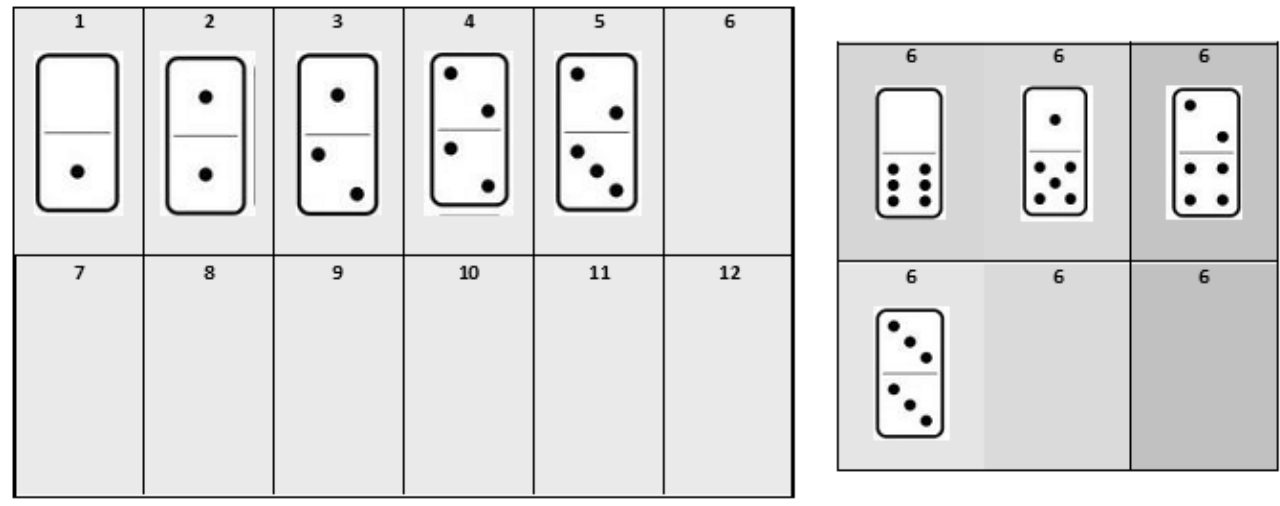

Figura 7-1 y 7-2. Ejemplo de tableros para realizar el juego: Cada coche en su lugar. 


\section{CONCLUSIONES E IMPLICACIONES}

La Música puede considerarse como arte, ciencia o como un lenguaje y no existe una definición única de tal concepto debido a las variaciones que la música experimenta con el paso del tiempo.

La Música contribuye a la formación integral de todas las facultades del alumno y no exclusivamente a las musicales, dado que los distintos aprendizajes y las diversas formas que tiene el alumno para acceder al conocimiento, así como la dimensión afectiva, cognitiva y psicomotriz pueden mejorar de forma destacable si éste se inicia de forma temprana en la educación musical.

Se han recopilado un total de 98 canciones para las etapas de El y EP, clasificadas por categorías con letra y partitura sobre contenidos matemáticos cuyas fichas pueden ser consultadas en la página web ya descrita. Esta guía de canciones matemáticas constituye un importante material para los docentes que imparten estas enseñanzas, permitiendo que se vea favorecida la construcción de conocimientos matemáticos mediante la música como una actividad lúdica y motivadora para los alumnos en consonancia con Casas, 2001, Pascual, 2006 y Levitin, 2011.

Los ejemplos nos muestran cómo puede integrarse la canción dentro del contenido y las actividades que son las tareas diarias del aula, así pues las canciones no son solamente un entretenimiento lúdico sino también un recurso de apoyo muy válido para la enseñanza en estas etapas (Casals, Carrillo y González, 2014, Alsina, 2012; Iturbe, 2012; Novo, 2013; Chao, Mato y López 2015)

El docente puede completar la ficha de las canciones con grabaciones y videoclips, de producción propia, para así obtener junto con la guía un documento auditivo y visual. Esta ampliación aportará nuevos contenidos y actividades para trabajar las canciones en la escuela como las actividades que proponen de Mato, Chao, y Chao, (2019).

La investigación es lo suficientemente abierta para que el docente la amplíe a otras materias y a otras etapas educativas e incluso pueda comprobar, de una manera práctica, la eficacia de dichas canciones en el aula.

\section{REFERENCIAS BIBLIOGRÁFICAS}

Alsina, Á. (2012) Hacia un enfoque globalizado de la educación matemática en las primeras edades. Números. Revista de didáctica de las matemáticas, 80, 7-24.

Aznárez, J.J.(1992) Nuestras canciones 1, 2. Canciones infantiles para una Educación Musical natural. Pamplona. Ediciones "Serie música para todos".

Barrantes, M. y Barrantes, M.C. (2017). Geometría en la Educación Primaria. Badajoz. Ed. Indugrafic digital. Bernal, J., Calvo, M. L. y Martín, C. (2000) Repertorio de canciones para la Educación Infantil. Málaga. Aljibe.

Canals, M. A. (2001). Vivir las matemáticas. Barcelona: Octaedro - Rosa Sensat.

Casas, M. V. (2001) ¿Por qué los niños deben aprender música? Colombia médica, 32 (4), 197-204.

Casals, A., Carrillo, C., González - Martín, C. (2014). La música también cuenta: combinando matemáticas y música en el aula. Revista Electrónica de Música en la Educación, 34, 1-17. Recuperado de: https://ojs.uv.es/index.php/LEEME/article/view/9861

Chao, R., Mato, M. D., y López, A. M. (2015) ¿Se trabajan de forma interdisciplinar música y matemáticas en educación infantil? Educaçao e pesquisa, 41 (4), 1009-1022.

Hernández, E. (1984). Pensar y jugar hablar y escuchar: guía didáctica para niños de 3-6 años. Barcelona. Ediciones Paulinas.

Hughes, J. (2014). Creative experiences in an interdisciplinary honors course on mathematics

in music. Journal of Mathematics and Music, 8 (2), 131-143. Recuperado de https://doi.org/10.1080/

17459737.2014.936916

Campo Abierto, v. 40, n. 1, p. 103-118, 2021 
Iturbe, B. (2012) Música y competencias básicas: competencia matemática. Padres y Maestros/Journal of Parents and Teachers, 345, 1-4.

Lesser L.M. (2000). Sum of songs: making mathematics less monotone! Math Teach, 93, 372-7

Lesser, L.M. (2001). Musical Means: Using Songs in Teaching Statistics, Teaching Statistics, 23(3), 81-85

Levitin, D. J. (2011) Tu cerebro y la música. Barcelona. RBA.

Mato, D. y Muñoz,J. M. (2010). Efectos generales de las variables actitud y ansiedad sobre el rendimiento en matemáticas en alumnos de educación segundaria obligatoria. Implicaciones para la práctica educativa. Revista de Ciencias Psicológicas, 4 (1), 27-40. Recuperado de https: //doi.org/10.22235/cp.v4i1.109

Mato,D; Chao,R. y Chao, A (2019). Efecto de enseñar matemáticas a través de actividades musicales. Relime, Vol. 22 (2), 163-184.

Montiel, M. (2017). Un experimento piloto sobre la enseñanza interdisciplinaria integrada a nivel universitario: matemáticas y música. Foro de Educación, 15(22), 1-30.

Noll, T. (2014). Getting Involved with Mathematical Music Theory. Journal of Mathematics and Music,8(2), 167-182. Recuperado de https://doi.org/10.1080/17459737.2014.943818

Novo, M. L. (2013) Creciendo con las matemáticas. Ponencia en XVI JAEM Palma 2013: Jornadas sobre el Aprendizaje y la Enseñanza de las Matemáticas. Julio 2013 (1-18) Consultado el 15 de abril del 2018 en: https://www.researchgate.net

Pascual, P. (2006) Didáctica de la música Para Educación Infantil. Madrid. Pearson Educación.

Robertson. W y Lesser L.M. (2013) Scientific skateboarding and mathematical music: edutainment that actively engages middle school student. European Journal of Science and Mathematics Education Vol. 1 (2), 60-68.

Rodríguez, M. E. (2011) La matemática y su relación con las ciencias como recurso pedagógico. Números. Revista de Didáctica de las Matemáticas, 77, 35-49.

San Andrés, C. (2000) Jugar, cantar y contar: juegos y canciones para los más pequeños. Madrid. Teleno.

Sanders, E. (2012). Investigating the relationship between musical training and mathematical thinking in children. Procedia-Social and Behavioral Sciences, 55, 1134-1143. Recuperado en: https://doi.org/ 10.1016/j.sbspro.2012.09.607

Sanuy, C y Cortés, L. (1978) Enseñar a jugar: juegos educativos para el desarrollo de la personalidad. Madrid. Marsiega.

\section{Webgrafía}

La página web Guía de canciones matemáticas para Educación Infantil y Primaria, que contiene las 98 canciones de nuestra guía. Recuperado de http://guiadecancionesmatematicas.piojosoft.es/

Bensaya, P. Presencias de Música. Recuperado de: www.presencias.net

Canciones Infantiles. Recuperado de: http://canciones-infantiles.com.es/

Dany Rosevear. La pájara pinta. Recuperado de: http://www.singinggamesforchildren.com/A\%20Cluster\%202.5\%20Spanish/6673\%20Spanish\%20s ongs\%20w.htm

Descubriendo la Música. Recuperado de: http://www.descubriendolamusica.com/

Geocites.Recuperado de: http://www.geocities.ws/ishar20/canciones partituras.html

Guía de canciones matemáticas para Educación Infantil y Primaria. Recuperado de: http://quiadecancionesmatematicas.piojosoft.es/

Isuuu. Guía Didáctica: Las Figuras geométricas. Recuperado de: http://issuu.com/caracol012/docs/guia didactica las figuras geometricas

Lets play kids music. Recuperado de: https://www.letsplaykidsmusic.com/

Mamá Lisa's World. Recuperado de: https://www.mamalisa.com/?t=hubsh

Mother Goose Club. Recuperado de: https://www.mothergooseclub.com/

Piano lessons for children. Recuperado de: http://www.pianolessons4children.com/songs/

Singing Games for Children. Recuperado de: http://www.singinggamesforchildren.com/index.htm

Super Simple.Recuperado de: https://supersimpleonline.com 